\title{
Symptom score questionnaire for nasolacrimal duct obstruction in adults - a novel tool to assess the outcome after endoscopic dacryocystorhinostomy*
}

\author{
Grigori Smirnov ${ }^{1}$, Henri Tuomilehto ${ }^{1}$, Hannu Kokki ${ }^{2}$, Tatu Kemppainen ${ }^{1}$, \\ Vesa Kiviniemi ${ }^{3}$, Juhani Nuutinen ${ }^{1}$, Kai Kaarniranta ${ }^{4,5}$, Juha Seppä ${ }^{1}$ \\ 1 Department of Otorhinolaryngology, Institute of Clinical Medicine, Kuopio University Hospital, and \\ University of Eastern Finland, Finland \\ 2 Department of Anesthesiology and Intensive Care, Institute of Clinical Medicine, Kuopio University Hospital \\ and University of Eastern Finland, Finland \\ 3 IT Centre, University of Eastern Finland, Finland \\ 4 Department of Ophthalmology, Institute of Clinical Medicine, Kuopio University Hospital, Finland \\ 5 Department of Ophthalmology, Institute of Clinical Medicine, University of Eastern Finland, Finland
}

SUMMARY

\begin{abstract}
Background: The incidence of nasolacrimal pathway obstruction increases with age, and dacryocystorhinostomy (DCR) is a commonly applied surgical technique to treat severe cases. However, no disease-specific tools to assess the symptoms and the subjective outcome after DCR have been established. We have developed a specific Nasolacrimal Duct Obstruction Symptom Score (NLDO-SS) questionnaire to evaluate the outcome, and tested it in a prospective clinical trial.

Methods: Sixty-eight consecutive primary endoscopic dacryocystorhinostomy (EN-DCR) procedures were performed in 64 patients during 2004-2008. Preoperatively and during the three follow-up visits, the patients filled in the NLDO-SS, and at the second and third follow-up visits they also filled in the Glasgow Benefit Inventory (GBI) questionnaire. At one year after the operation, a GBI questionnaire was sent to the patients.

Results: The surgical success rate of EN-DCR was 93\%. EN-DCR resulted in a significant reduction in all of the eight symptoms scores of the NLDO-S. The GBI scores indicated a benefit at 2 months and an even higher benefit at 6 months, but no further improvement was found between 6 and 12 months. The correlation between the total GBI and NLDO-SS was significant.

Conclusions: EN-DCR improves the quality of life as measured by the GBI. The NLDO-SS correlated with the GBI and gave more information about the benefits after EN-DCR than GBI alone. The NLDO-SS proved to be an effective tool to evaluate lacrimal obstructions and ENDCR benefits. Further studies to validate NLDO-SS are needed.
\end{abstract}

Key words: endoscopic dacryocystorhinostomy, assessment, symptom score, quality of life

\section{INTRODUCTION}

Nasolacrimal duct obstruction (NLDO) is a relatively common condition in elderly patients ${ }^{(1)}$. The irritating symptoms related to NLDO, such as tearing, blurred vision, pain around the eye, and recurrent infections in the lacrimal sac, may significantly deteriorate individuals' quality of life (QoL).

Dacryocystorhinostomy (DCR) is indicated in patients whose NLDO symptoms and signs are not relieved with conservative treatment. The aim of DCR is to create a constant rhinostoma between the medial wall of the lacrimal sac and the mucosa of the nasal cavity. External surgery has been the standard approach for DCR, but over the last two decades, the minimal invasive endoscopic technique (EN-DCR) has become accepted as a valid treatment to bypass both saccal and post-saccal obstruction of the lacrimal pathway ${ }^{(2)}$.

Patient satisfaction through relieved symptoms and improved QoL are the predominant considerations determining the success of surgery. Previous studies indicate that DCR relieves the symptoms and consequently improves the health status and general well being of patients suffering from $\mathrm{NLDO}^{(3)}$.

The Glasgow Benefit Inventory (GBI) is a generic validated 18- 
item measure of patient benefits developed for otolaryngological interventions ${ }^{(4)}$ and it has been used to evaluate patient perception of benefits from the different DCR techniques ${ }^{(5)}$. In previous studies, postoperative QoL assessments after DCR have been done using mailed questionnaires, and the time of the single assessment has varied from 6 to 37 months after the procedures ${ }^{(5-7)}$. However, there are no specific validated measures designed to assess the subjective outcome after DCR, and neither has the optimal time for assessment been established. Moreover, the GBI is not specifically developed for surgery in patients with ocular symptoms.

To improve the outcome evaluation, we developed a simple Nasolacrimal Duct Obstruction Symptom Score questionnaire (NLDO-SS) with items focused on the common ocular and nasal symptoms of NLDO. The specific aims of the present study were to evaluate the impact of primary EN-DCR on the QoL and symptoms, and the correlations between GBI, NLDO-SS and objective findings after the surgery.

\section{MATERIALS AND METHODS}

Study design

This study is a part of our prospective EN-DCR trial, and some results have already been published ${ }^{(8-10)}$. The recruitment started in fall 2004, and ended in 2008. The participants were consecutively recruited from the patients referred to the outpatient clinic of the Department of Otorhinolaryngology at Kuopio University Hospital, in Kuopio, Finland. The patients were eligible for participation if they were adults (age 18 years or older), were American Society of Anesthesiologist physical status I - III, and were scheduled for primary lacrimal pathway surgery due to saccal or post-saccal NLDO. Exclusion criteria were pre-saccal obstruction, previous nasolacrimal surgery, malignancy in the paranasal sinuses, nasal cavity, or lacrimal pathway, mental disability, pregnancy or breast feeding. The study protocol was approved by the Research Ethics Committee of the Hospital District of Northern Savo, Kuopio, and it has been registered in the ClinicalTrials.gov (ID: NCT00571129). The patients were given oral and written information about the trial protocol and they provided written consent.

All patients made a preoperative visit and three postoperative visits: at one week, and at 2 and 6 months after the surgery. At 12 months, they were sent a GBI questionnaire to be returned in a prepaid envelope. The study flow-chart is presented in Figure 1.

\section{Preoperative assessment}

The clinical assessment for all patients was done by an otorhinolaryngologist and included endoscopic examination of the nasal cavity and lacrimal sac irrigation. Findings in the nasal cavity were scored using the Lund-MacKay endoscopic score (11). Each patient had a preoperative paranasal computed tomography scan.

\section{Operative technique}

A standardized general endotracheal anesthesia technique was used for all patients. The operations were performed by three otorhinolaryngologists (J.S., H.T., G.S.) using the same surgical endoscopic powered instrumentation technique, which is described in detail in our previous publication ${ }^{(8)}$.

\section{Patients}

Sixty-five consecutive patients were asked to participate, of whom 64 agreed. Four patients underwent bilateral surgery at the same operative session, and thus the study involved 68 consecutive primary EN-DCR procedures for 64 patients suffering from dacryostenosis, or recurrent or chronic dacryocystitis. The baseline demographics of the patients are presented in Table 1.

Table 1. The baseline demographics of the patient.

\begin{tabular}{ll} 
Male & 15 \\
Female & 49 \\
Age: Mean (SD) & $63(13)$ \\
Diagnoses & \\
Dacryostenosis & 31 \\
Recurrent/chronic dacryocystitis & 37 \\
Laterality & \\
Right & 36 \\
Left & 32 \\
\hline
\end{tabular}

Data are the mean $(\mathrm{SD})$ or number of cases. $\mathrm{SD}=$ Standard Deviation.

Table 2. Nasolacrimal Duct Obstruction Symptom Score questionnaire (NLDO-SS)

Symptom
Tearing
Discharge in the eye
Swelling around the eye
Pain around the eye
Change in visual acuity
Nose blockage
Nasal cavity discharge
General condition
Total score

Numeric Rating Scale (NRS; $0=$ no symptom, $10=$ worst imaginable symptom)

\section{Questionnaires}

During the preoperative visit and at each of the three postoperative visits, the patients filled out an NLDO-SS questionnaire that was developed by the authors to improve the outcome evaluation of patients undergoing EN-DCR. The NLDO-SS questionnaire consists of five items focused on the common ocular symptoms of NLDO, two items describing the conditions in the nasal cavity, and one item on the general condition (Table 2). In the NLDO-SS, the symptoms are graded using an 11-point numeric rating scale (NRS; $0=$ no symptom, $10=$ worst imaginable symptom). The total number of points for NLDO-SS ranges from 0 to 80 points. The patients filled in the 
NLDO-SS by themselves during the visit, before the clinical examination.

The patients completed the GBI at two postoperative visits, at 2 and 6 months, and it was posted to them with a covering letter 12 months after the surgery to be returned in an enclosed prepaid envelope. The GBI consists of 18 questions, which in the present study were adapted to be used for a nasal operation. In the GBI, the response to each question is based on a five-point Likert scale ${ }^{(12)}$ ranging from a large deterioration in health status through to a large improvement in health status. The GBI total score consists of three subscales, each ranging from -100 (maximal negative benefit) through zero (no changes) to +100 (maximal positive benefit). The subscales consist of a general subscale (12 questions), a social support subscale (three questions), and a physical health subscale (three questions) ${ }^{(4)}$.

\section{Postoperative care and assessment}

In addition to the preoperative clinical examination before the surgery, the patients had clinical examinations at the three follow-up visits. The objective assessment was performed by an otorhinolaryngologist using a rigid nasoendoscope and lacrimal sac irrigation at each visit. The extent of mucosal edema, polyposis, crusting, secretions and scarring was assessed and scored using the Lund-MacKay endoscopic score ${ }^{(11)}$. The surgical outcome was considered successful if the saline solution freely

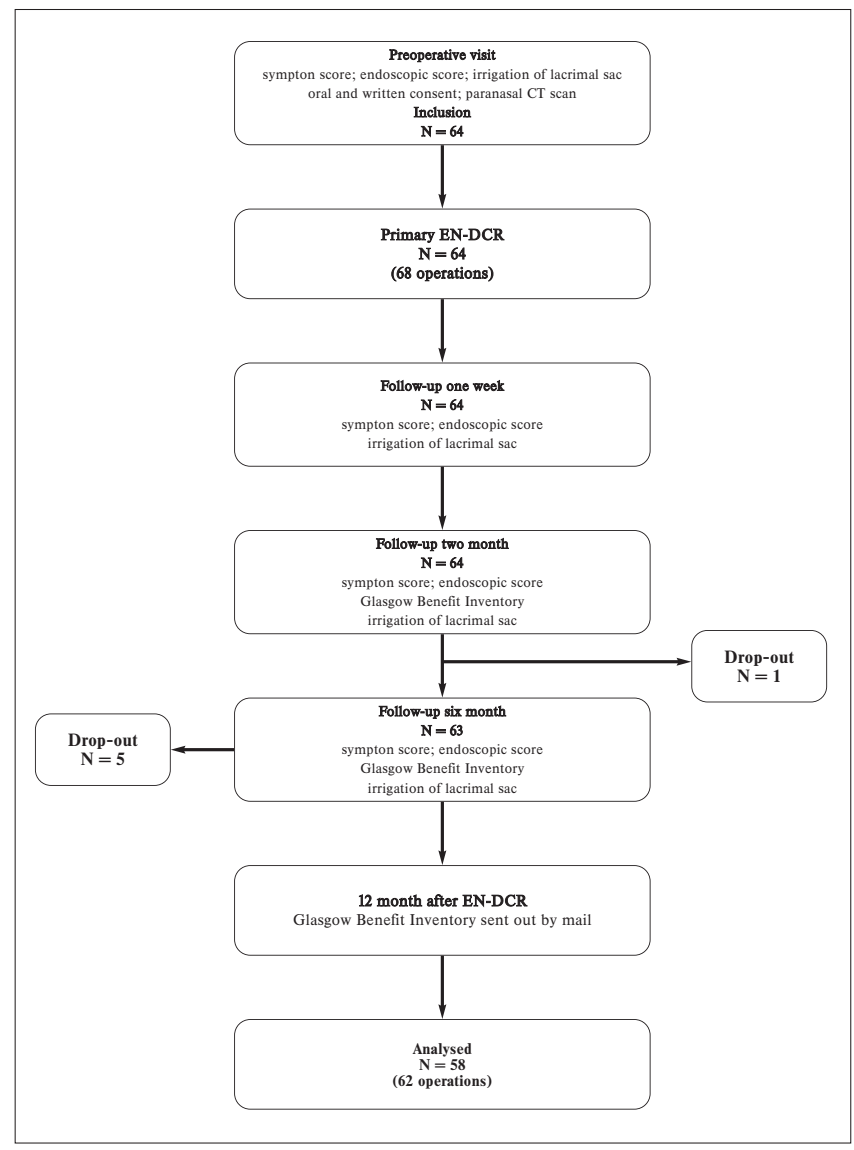

Figure 1. Study flowchart $(\mathrm{N}=$ number of patient). reached the nose during the lacrimal sac irrigation and if the patients had no tearing, or recurrent conjunctival discharge.

\section{Statistics}

Patient characteristics and variables were analyzed with the Statistical Package for Social Sciences (SPSS software version 17.0 for Windows, SPSS Inc., Chicago, IL, USA). Repeated measures analysis of variance between groups was used to study the differences between the measurement time points. Post hoc tests were based on estimated marginal means and were Bonferroni corrected. Correlations between variables were assessed with Spearman's correlation coefficient. A 95\% confidence interval was calculated for the main results. All the analyses were performed on an intent-to-treat basis. Differences were regarded as statistically significant if a twosided p-value was less than 0.05. Data are expressed as the number of cases or mean with standard deviation (SD).

\section{RESULTS}

\section{Surgical outcome}

All 64 patients presented at the one week and 2-month followup visits. One patient died four months after the operation, and thus 63 patients presented at the 6-months follow-up. The 12-months questionnaire was returned by 58 patients. Additional surgery was performed in five patients due to abnormalities in the nasal cavity interfering with the operation, such as septal deviation or hypertrophic middle turbinate. Uncinectomy was performed in two patients with recurrent or chronic sinusitis at the same time as EN-DCR. All the patients were discharged from the hospital on the first postoperative day, and no intra-operative or immediate postoperative complications likely to affect the study results occurred during the study. The overall surgical success rate after primary EN-DCR at the 6-month follow-up was $93 \%$.

\section{Objective assessment (Lund-MacKay Endoscopic Score)}

As was to be expected, due to crusting and secretions related to the immediate operation we found a statistically significant increase in one-week postoperative Lund-MacKay Endoscopic score compared with the preoperative score $(\mathrm{p}=0.001)$. There were no significant differences between the preoperative and the 2- or 6-month follow-up visit findings in the Lund-MacKay Endoscopic Score $(\mathrm{p}=0.17)$.

\section{GBI}

All three follow-up questionnaires were fully completed by 58 of $63(92 \%)$ of the patients (Figure 1). A benefit from the ENDCR was observed at all three times of assessment. The patients reported a marked benefit already at 2 months (mean GBI total score + 37 (SD: 28)), and it had improved significantly at 6 months (mean GBI total score +52 (29): mean difference $15,95 \%$ CI 6-24, $\mathrm{p}=0.001$ ). At 2 months, the GBI scores were positive in all three subsets, and between the 2- and 6months assessments a further gain was reported in general and 


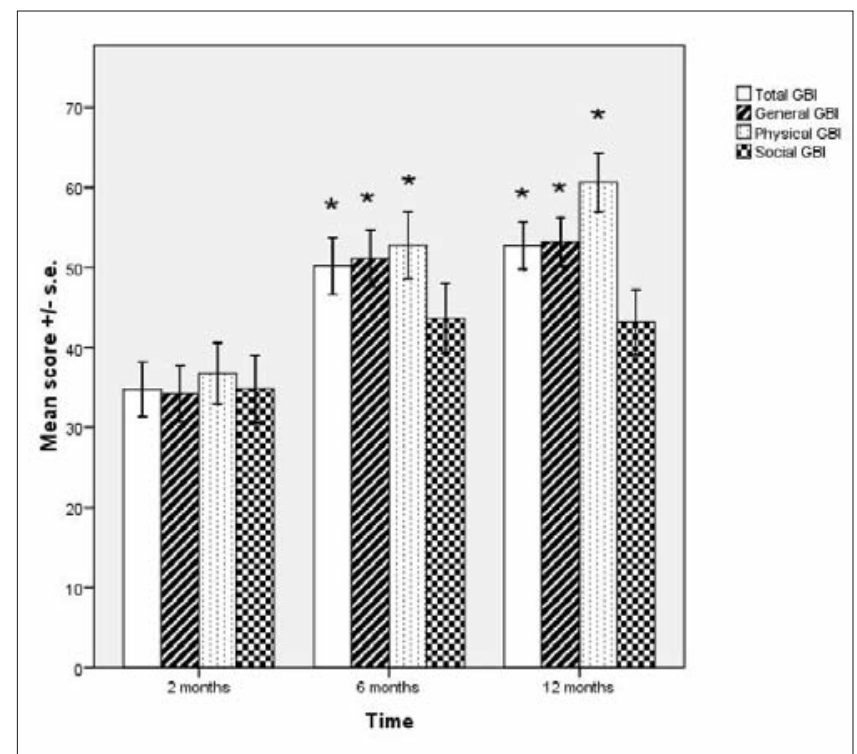

Figure 2. Distribution of GBI scores by subsets at three assessment times after primary EN-DRC.

Data are mean with standard error. (s.e.).

* Statistically significant difference, $\mathrm{P}=0.001$

GBI $=$ Glasgow Benefit Inventory

EN-DCR $=$ endoscopic dacryocystorhinostomy

physical subsets $(\mathrm{p}=0.001)$. No significant differences were found between the 6 and 12 months GBI scores in any subset (Figure 2).

\section{Nasolacrimal Duct Obstruction Symptom Score (NLDO-SS)}

EN-DCR resulted in a significant reduction in all of the eight symptom scores: the mean total NLDO-SS fell from 38 (SD; 13 ) at baseline to 10 (10) points (mean difference 28, 95\% CI: $23-33, \mathrm{p}=0.001$ ) at 6 months (Figure 3 ). The greatest reduction was detected in the five ocular symptoms, in which the mean score decreased from 26 (9) at baseline to 5 (7) points $(\mathrm{p}=0.001)$ at 6 months (Figure 4).

GBI, Nasolacrimal Duct Obstruction Symptom Score and Endoscopic Lund-MacKay Score correlations

The correlations were calculated for the 2- and 6-month's postoperative visits. There was a negative correlation between total GBI and total NLDO-SS $(\mathrm{r}=-0.314, \mathrm{p}=0.009 ; \mathrm{r}=-0.394, \mathrm{p}=$

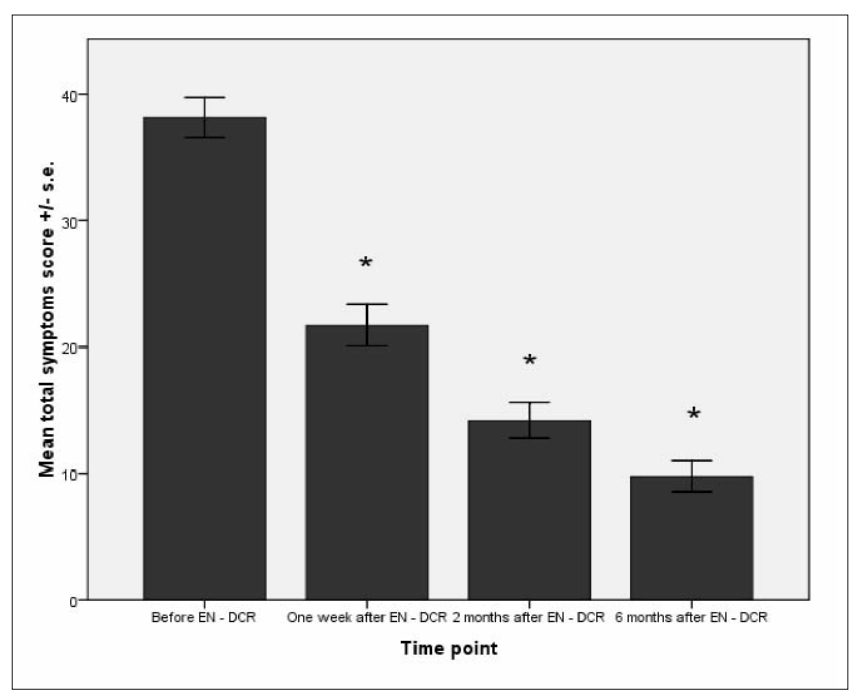

Figure 3. Total Nasolacrimal Duct Obstruction Symptomen Score (NLDO-SS) at baseline and three assessment times after primary EN-DCR.

Data are mean with standard error. (s.e.).

* Statistically significant difference, $\mathrm{P}=0.001$

EN-DCR $=$ endoscopic dacryocystorhinostomy

0.001, respectively), indicating good consistency of the two scoring systems. Moreover, a negative correlation was found between total GBI and the Endoscopic Lund-MacKay Score at 6 months after operation $(\mathrm{r}=-0.258, \mathrm{p}=0.037)$, and a positive correlation between Endoscopic Lund-MacKay Score and total NLDO-SS for the 2- and 6-month's postoperative visits $(\mathrm{r}=0.289, \mathrm{p}=0.017 ; \mathrm{r}=0.245, \mathrm{p}=0.046$, respectively) (Table 3 ).

\section{DISCUSSION}

The surgical success rate in the present study was $93 \%$ at 6 months, which is similar to that reported in previous studies with primary EN-DCR ${ }^{(2)}$. However, although surgical success is the principal objective parameter in surgical outcome assessment, from the patients' perspective the relief of symptoms and improvement in well-being are the paramount expectations. To provide accurate information related to health benefits in adults, there is a need for assessment tools addressing symptomatology of the NLDO. There is a validated symptom

Table 3. Correlation between Glasgow Benefit Inventory, Nasolacrimal Duct Obstruction Symptom Score (NLDO-SS) and Endoscopic Lund-MacKay Score at two and six months after EN-DCR.

\begin{tabular}{|c|c|c|c|c|}
\hline & \multicolumn{2}{|c|}{$\begin{array}{l}\text { At two months } \\
\text { after EN-DCR }\end{array}$} & \multicolumn{2}{|c|}{$\begin{array}{l}\text { At six months } \\
\text { after EN-DCR }\end{array}$} \\
\hline & r-value & p-value & r-value & p-value \\
\hline \multicolumn{5}{|l|}{ Variable } \\
\hline Total GBI score $<=>$ NLDO-SS & $-0.314 * *$ & 0.009 & $-0.394 * *$ & 0.001 \\
\hline Lund-MacKay score $<=>$ NLDO-SS & $0.289^{*}$ & 0.017 & 0.245 & 0.046 \\
\hline Total GBI score $<=>$ Lund-MacKay score & 0.658 & 0.055 & $-0.258^{*}$ & 0.037 \\
\hline $\mathrm{r}$ - correlation coefficient & \multicolumn{4}{|c|}{ * Correlation is significant at the 0.05 level (2-tailed) } \\
\hline ** Correlation is significant at the 0.01 level (2-tailed) & \multicolumn{4}{|c|}{ EN-DCR $=$ endoscopic dacryocystorhinostomy } \\
\hline
\end{tabular}




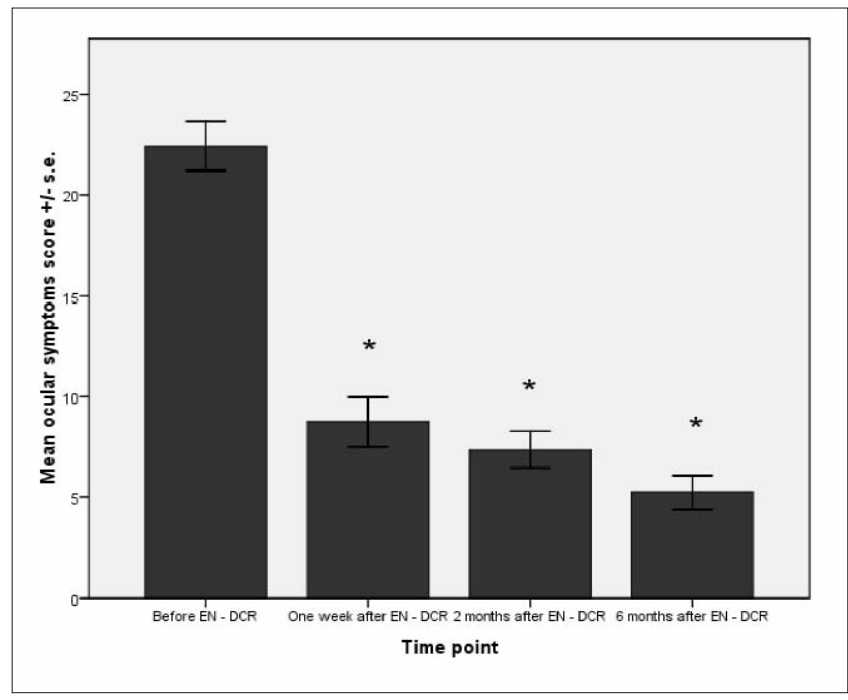

Figure 4. The mean scores of five ocular symptoms at baseline and three assessment times after primary EN-DCR.

Data are mean with standard error. (s.e.).

* Statistically significant difference, $\mathrm{P}=0.001$

EN-DCR = endoscopic dacryocystorhinostomy

questionnaire for NLDO for children but not for adults ${ }^{(13)}$. This is why we developed an ocular and nasal symptom questionnaire for the pre- and postoperative subjective assessment for the adult patients with NLDO.

Successful surgical outcome does not necessarily confer an improvement in $\mathrm{QoL}^{\left({ }^{(6)}\right.}$. To estimate the outcome and health benefit, reliable assessment tools are needed to confirm the changes in symptoms and patients' well-being after the intervention. The GBI is commonly used for the measurement of surgical benefit in rhinological procedures ${ }^{(14)}$, and it has also been used in patients with EN-DCR ${ }^{(5-7)}$. The main limitation of the GBI is that it is adapted for nasal surgery, but not for nasolacrimal surgery. EN-DCR operations are performed endonasally, whereas the main causes of complaints are in the ocular region. Bakri and co-authors ${ }^{(5)}$ used a GBI questionnaire, and showed that the GBI is a useful tool to compare and evaluate outcome, but it is not able to reflect "subtle changes in quality of life" after two different DCR techniques.

In the present study, both questionnaires were found to reliably measure the outcome of EN-DCR, and a statistically significant correlation between the GBI and NLDO-SS questionnaire was observed (Table 3). However, GBI alone is not able to distinguish the QoL factors, which are connected to symptom changes. On the other hand, the NLDO-SS questionnaire proved to be a simple and sensitive tool to assess disease-specific symptoms changes after EN-DCR.

Our study shows that successful EN-DCR has a significant impact on the patient QoL as measured by GBI. Moreover, the health benefits improved significantly during the first six months follow-up period. However, after the 6-months assess- ment, no further improvement was detected (Figure 2). When we compared the 12-months GBI with the GBI at 6 months, an inconsistency was detected in some elderly patients' scores: the scores for most questions were lower at 12 months than at 6 months. These inconsistencies were the reason why we recalled 11 patients for an extra follow-up visit at 12 months. During this additional visit, the patients filled in an NLDO-SS questionnaire before the objective examination, which was performed using a rigid endoscope and lacrimal sac irrigation. In 2 out of these 11 cases, scar tissue had formed at the rhinostomy site. In the 9 other cases, the inconsistency in the GBI scores was due to misunderstanding of some GBI questions by the patients. Based on the NLDO-SS and objective findings, the rhinostomy was open and these 9 patients had no complaints. The incidence of nasolacrimal duct obstruction is $20 / 100,000$ and it increases with age ${ }^{(1)}$. In the present study, the mean age of patients at the time of the surgery was 63 years. It was observed that the 18 -item GBI can be difficult for some of the elderly patients to complete by themselves without any guidance. Therefore, to obtain reliable information on patients' well-being, it is advisable to develop and use simplified questionnaires, such as the NLDO-SS, which was found easy to understand and, most importantly, gave information consistent with objective findings.

Postoperative care and the timing of the postoperative followup visits are important for optimal outcomes ${ }^{(9)}$. Any disturbance in the normal healing process endangers the surgical outcome of EN-DCR and may lead to postoperative infection, granulation tissue and scar formation at the rhinostomy site ${ }^{(15-}$ 17). In this prospective study, the patients went through three follow-up visits: at one week, and at 2 and 6 months after operation. Even so, 2 patients developed obstruction between the 6 and 12 months after the EN-DCR. Thus, successful outcome at 6 months after EN-DCR did not assure success. Our results indicate that a follow-up beyond 6 months after EN-DCR may detect some late complications.

\section{CONCLUSION}

This study shows that in adult EN-DCR patients, a postoperative follow-up lasting longer than 6 months may be needed. Furthermore, the present study indicates that the NLDO-SS questionnaire is a suitable tool for subjective postoperative outcome assessment after EN-DCR, and provides more information than the GBI. Although these results are promising, further prospective studies are needed to confirm and validate the usefulness of the NLDO-SS.

\section{ACKNOWLEDGEMENTS}

This study was not financially supported from external sources.

\section{CONFLICT OF INTEREST \\ None}

Trial Registration clinicaltrials.gov ID: NCT00571129 


\section{REFERENCES}

1. Woog JJ. The incidence of symptomatic acquired lacrimal outflow obstruction among residents of Olmsted County, Minnesota, 19762000 (an American Ophthalmological Society thesis). Trans Am Ophthalmol Soc. 2007; 105: 649-666.

2. Sprekelsen MB, Barberan MT. Endoscopic dacryocystorhinostomy: surgical technique and results. Laryngoscope 1996; 106 (2 Pt 1): 187-189

3. Cokkeser Y, Evereklioglu C, Er H. Comparative external versus endoscopic dacryocystorhinostomy: results in 115 patients (130 eyes). Otolaryngol Head Neck Surg. 2000;123: 488-491.

4. Robinson K, Gatehouse S, Browning GG. Measuring patient benefit from otorhinolaryngological surgery and therapy. Ann Otol Rhinol Laryngol. 1996; 105: 415-422.

5. Bakri SJ, Carney AS, Robinson K, et al. Quality of life outcomes following dacryocystorhinostomy: external and endonasal laser techniques compared. Orbit 1999; 18: 83-88

6. Ho A, Sachidananda R, Carrie S, et al. Quality of life assessment after non-laser endonasal dacryocystorhinostomy. Clin Otolaryngol. 2006; 31: 399-403.

7. Spielmann PM, Hathorn I, Ahsan F, et al. The impact of endonasal dacryocystorhinostomy (DCR), on patient health status as assessed by the Glasgow benefit inventory. Rhinology 2009; 47: 48-50.

8. Smirnov G, Tuomilehto H, Terasvirta M, et al. Silicone tubing after endoscopic dacryocystorhinostomy: is it necessary? Am J Rhinol. 2006; 20: 600-602.

9. Smirnov G, Tuomilehto $\mathrm{H}$, Terasvirta $\mathrm{M}$, et al. Silicone tubing is not necessary after primary endoscopic dacryocystorhinostomy: a prospective randomized study. Am J Rhinol. 2008; 22: 214-217.

10. Smirnov G, Pirinen R, Tuomilehto H, et al. Strong expression of HSP47 in metaplastic nasal mucosa may predict a poor outcome after primary endoscopic dacryocystorhinostomy: a prospective study. Acta Ophthalmol. 2009;24.
11. Lund VJ, Mackay IS. Staging in rhinosinusitus. Rhinology 1993; 31: 183-184.

12. Likert R. A technique for the measurement of attitudes. Arch Psychol. 1932; 22: 1-55.

13. Holmes JM, Leske DA, Cole SR, et al. Nasolacrimal Duct Obstruction Questionnaire Study Group, et al. A symptom survey and quality of life questionnaire for nasolacrimal duct obstruction in children. Ophthalmology 2006; 113: 1675-1680.

14. Chester AC, Sindwani R. Symptom outcomes in endoscopic sinus surgery: a systematic review of measurement methods. Laryngoscope 2007; 117: 2239-2243.

15. Osguthorpe JD, Calcaterra TC. Nasolacrimal obstruction after maxillary sinus and rhinoplastic surgery. Arch Otolaryngol. 1979; 105: 264-266.

16. Osguthorpe JD, Hoang G. Nasolacrimal injuries. Evaluation and management. Otolaryngol Clin North Am. 1991; 24: 59-78.

17. Allen K, Berlin AJ. Dacryocystorhinostomy failure: association with nasolacrimal silicone intubation. Ophthalmic Surg. 1989; 20: 486-489.

Grigori Smirnov, MD

Department of Otorhinolaryngology

Institute of Clinical Medicine

Kuopio University Hospital

P.O. Box 1777

FI -70211 Kuopio

Finland

Tel : $+358-17-173311$

Fax : +358-17-172509

E-mail : grigori.smirnov@kuh.fi 